\title{
Vertical distribution of clouds over Hampton, Virginia observed by lidar under the ECLIPS and FIRE ETO programs
}

\author{
D.M. Winker ${ }^{\mathrm{a}}$, M.A. Vaughan ${ }^{\mathrm{b}}$ \\ a Atmospheric Sciences Division, NASA Langley Research Center, Hampton, IA 23681, CSA \\ 'Science Applications International Corporation, I Enterprise Parkway, Hampton, WA 23666. LiS.
}

(Received January 21, 1993; revised and accepted June 15, 1993)

\begin{abstract}
Intensive cloud lidar observations have been made by NASA Langley Research Center during the two observation phases of the ECLIPS project. Less intensive but longer term observations have been conducted as part of the FIRE extended time observation (ETO) program since 1987 . We present a preliminary analysis of the vertical distribution of clouds based on these observations. A mean cirrus thickness of just under $1 \mathrm{~km}$ has been observed with a mean altitude of about $80 \%$ of the tropopause height. Based on the lidar data, cirrus coverage was estimated to be just under $20 \%$, representing roughly $50 \%$ of all clouds studied. Cirrus was observed to have less seasonal variation than lower clouds. Mid- level clouds are found to occur primarily in association with frontal activity.
\end{abstract}

\section{Introduction}

It has been recognized that clouds play an important and not well understood role in climate and climate change (Ramanathan et al., 1989). Reliable and accurate climatologies of even simple cloud properties such as base and top heights are not well established, particularly for cirrus. Traditionally, the vertical distributions of clouds have been estimated either by ground observers (Hahn et al., 1982,1984 ) or by aircraft observers, occasionally in dedicated programs (deBary and Möller, 1963). More recently, techniques utilitizing passive satellite instruments have been developed (Wylie and Menzel, 1989; Minnis et al., 1990). The International Satellite Cloud Climatology Project (ISCCP) (Rossow and Schiffer, 1991) has been established to develop an improved global cloud climatology. The value of this project will be enhanced by comparison with indepen- 
dently derived climatologies, even if the climatologies are only local. Unlike many other techniques, ground based lidar allows continuous monitoring of clouds and provides direct and accurate height information, in addition to some optical and microphysical properties. While having the disadvantage of not being able to penetrate dense clouds, lidar can penetrate relatively thin lower layers to observed higher layers which would be hidden to a ground observer. Lidar is particularly well suited for studying cirrus and other optically thin clouds, which present difficult retrieval problems for satellite instruments. NASA Langley Research Center has conducted intensive lidar cloud observations as part of ECLIPS (the Experimental Cloud Lidar Pilot Study), an international effort involving 16 lidar groups from the U.S., Europe, Asia and Australia (WMO, 1988). Lidar cloud observations have also been made on a routine basis since 1987 as part of the FIRE ETO (First ISCCP Regional Experiment Extended Time Observation) program (Cox et al., 1987). Three other lidar sites, all located in the United States, have also been involved in the ETO program. Designed to complement the FIRE intensive field observation (IFO) campaigns, the purpose of the FIRE ETO program is to produce a set of long term cloud lidar observations. In both programs observations are coordinated with satellite overpasses, providing a dataset which may be used for comparison with satellite cloud retrievals. In particular, the programs are intended to contribute to the validation and interpretation of cloud data products produced by the ISCCP. The ECLIPS and FIRE ETO programs will provide information not readily deduced from passive satellite cloud retrievals: primarily cloud base height, and the vertical structure and optical depth of cirrus clouds. In addition, ECLIPS is intended to explore the feasibility of creating an international network to obtain climatologies of cloud base height and cloud optical properties from lidar observations. In this paper we present preliminary results on cloud vertical distribution over Hampton, Virginia based on observations conducted under the ECLIPS and FIRE ETO programs.

\section{Instrumentation and observations}

The observations reported here were made in Hampton, Virginia $\left(76.5^{\circ} \mathrm{W}\right.$, $37.0^{\circ} \mathrm{N}$ ). The lidar system used for the observations employs the $532 \mathrm{~nm}$ linearly polarized output of a doubled Nd:YAG laser, an 8 " Celestron receiving telescope, and polarization-sensitive receiving optics. Two photomultiplier tubes are used to simultaneously sample backscattered light polarized parallel and perpendicular to the transmitted beam. The signal is digitized at a resolution of either 6,15 or $30 \mathrm{~m}$ and with 8-bit resolution. The laser is operated at a pulse rate between 3 and $10 \mathrm{~Hz}$, with averaged parallel and perpendicular returns being recorded every $15 \mathrm{~s}$. Other system characteristics are given in Table 1 . Cloud base and top heights and profiles of attenuated scattering ratio (defined below) and depolarization within cloud layers are routinely derived from the recorded returns. The system is usually operated in a vertical-pointing mode, but can be tilted several degrees off-zenith. Regions of low depolarization, which may be due to either horizon- 
Table 1

Instrumentation used in the ECLIPS and FIRE ETO programs

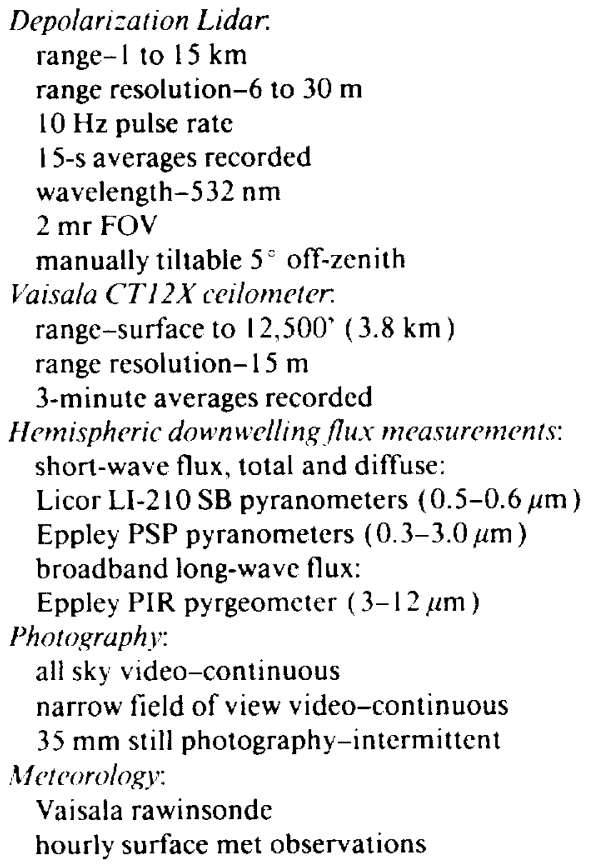

tally oriented plate crystals or supercooled water droplets, are sometimes seen within cirrus clouds. In these cases, the behavior of the depolarization signal when the system is tilted off-zenith allows discrimination between ice and water.

Lidar observations have been conducted in coordination with overpasses of the AVHRR instruments on the NOAA-10,11 and 12 satellites. For the last several years FIRE ETO observations have been made on a nominal schedule of two periods of 5 consecutive working days per month. Actual observations have averaged about 8 days per month. On observation days, observations are typically conducted coincident with one morning and one afternoon overpass occurring during normal working hours. A single observation lasts $1-2 \mathrm{~h}$, centered around the overpass time. Observations are not made during conditions of precipitation or fog, but otherwise are conducted whether conditions are clear or cloudy and regardless of cloud types present. The lidar cannot detect cloud bases under about $1 \mathrm{~km}$, but the lidar dataset is augmented by a ceilometer which detects cloud bases from about $3 \mathrm{~km}$ to the surface. The operating philosophy is that atmospheric conditions are to be randomly sampled so the dataset can be used to develop statistics of cloud occurrence.

ECLIPS activity has been concentrated in two intensive observation phases. At NASA Langley Research Center, observations for Phase I were conducted over 
the period from 15 October to 12 November, 1989, and for Phase II for 4 weeks in May 1991. Lidar observations were made continuously, except during periods of precipitation, over the 4 week period of Phase I. During Phase II, observations were conducted approximately $16 \mathrm{~h}$ per day, scheduled so that observations were made coincident with all AVHRR overpasses having zenith angles less than $60^{\circ}$. Results of depolarization observations are being compiled and will be reported at a later date.

Both ECLIPS and FIRE ETO lidar observations have been made in conjunction with ground-based measurements of shortwave and longwave fluxes. In addition, rawinsondes were launched from the lidar site during the two ECLIPS periods, coincident with about half the AVHRR overpasses, providing detailed profiles of temperature, humidity and winds. A variety of video and still photography of sky conditions is also available from the ECLIPS observation phases. Observer logbooks are maintained, recording WMO cloud clodes and other aspects of cloud appearance. Instrumentation used during ECLIPS and FIRE ETO is listed in Table 1.

\section{Method of analysis}

Fig. 1 shows lidar return signals typical of stratus or cumulus clouds. These cloud types typically exhibit high extinction and the lidar pulse is attenuated and disappears into the cloud in a few hundred meters, as in the cases shown here. Stratocumulus clouds often have significant horizontal structure and often the entire depth of the cloud layer can be penetrated in at least some areas of the cloud. Typical cirrus profiles are shown in Fig. 2. Cirrus clouds have low optical

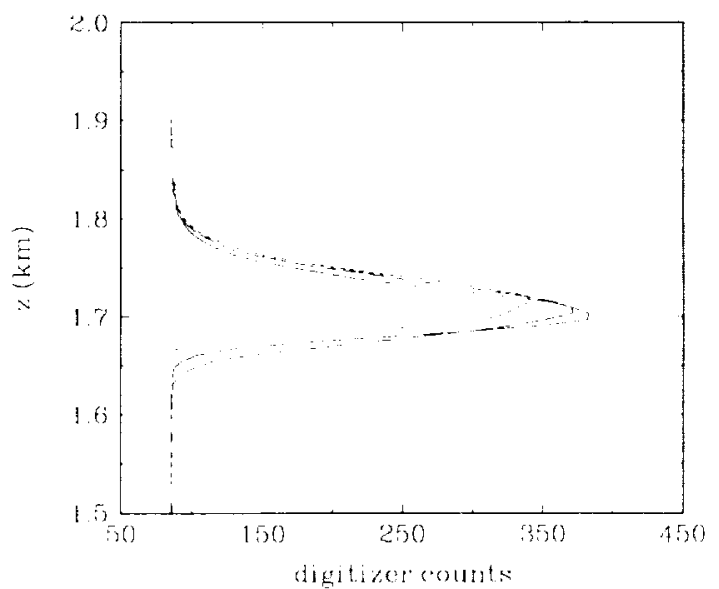

Fig. 1. Typical lidar signals from stratus clouds. Observations from the same cloud, separated by one minute. 


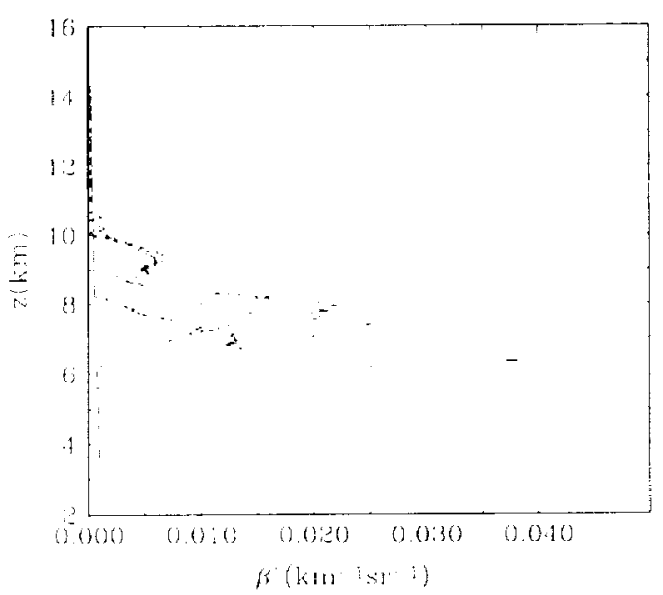

Fig. 2. Typical profiles of cirrus attenuated backscatter cross-section. Profiles from the same cloud system over a period of 20 minutes.

depths and the lidar pulse normally penetrates even very deep cirrus. Cirrus depths of greater than $4 \mathrm{~km}$ have been measured with the system.

Following Platt (1979) we write the lidar equation in the form:

$$
P(z)=\frac{C \beta(z)}{z^{2}} \exp \left[-2 \int_{0}^{z} \mathrm{~d} z \eta(z) \sigma(z)\right]
$$

which expresses the lidar return signal $P(z)$ as a function of altitude, $z$, in terms of the volume backscatter and volume extinction coefficients, $\beta(z)$ and $\sigma(z)$. $C$ is the lidar system constant and accounts for all of the factors necessary to convert the measured signal voltages into a calibrated return signal power, including the transmitted laser pulse energy. $\eta(z)$ is a factor accounting for contributions to the return signal from multiple scattering within clouds. $C$ may be determined by comparing the return signal from a clean region of the atmosphere with the return signal expected from a molecular atmosphere (Russell et al., 1979). The parameters $\beta(z), \sigma(z)$ and $\eta(z)$ are all unknowns. They may be lumped together and the lidar equation written in terms of an attenuated backscatter coefficient, $\beta^{\prime}(z)$ :

$$
P(z)=C \beta^{\prime}(z) / z^{2} \text {. }
$$

An attenuated scattering ratio may then be defined in terms of $\beta^{\prime}$ :

$$
S R^{\prime}=\beta^{\prime} / C \beta_{\mathrm{m}} \text {, }
$$

where $\beta_{m}^{\prime}$ is the attenuated backscatter cross-section of the molecular atmosphere:

$$
\beta_{\mathrm{m}}^{\prime}(\mathrm{z})=\beta_{\mathrm{m}}(\mathrm{z}) \exp \left[-2 \int_{0}^{z} d \mathrm{z} \sigma_{\mathrm{m}}(\mathrm{z})\right]
$$


- An attenuated scattering ratio profile can be constructed from a measured lidar return by dividing the normalized return profile by a molecular return profile computed using a model molecular atmosphere.

Cloud tops and bases are located by applying a threshhold algorithm to the profile of attenuated scattering ratio without actually solving the lidar equation for any of the unknowns. A range-dependent threshold is defined, based on the noise in the recorded lidar signal, and a signal excursion above the threshold value is identified as a cloud. Statistical smoothing is used to eliminate spurious cloud identifications due to noise spikes. Further details of the cloud-finding algorithm are given in the Appendix.

Except in cases of precipitation and blocking by lower layers, detection of cloud base is unambiguous. Detection of cloud top is not as straightforward. Cloud top is generally identified as the point where the return drops below the threshold value. If a lidar return is received from clear air above the cloud or from a higher cloud layer, the top of the lower cloud can be unambiguously identified and this is called the "true cloud top". If there is no lidar signal detected from above the cloud it cannot be determined if the lidar signal was completely attenuated inside the cloud, or if it was only attenuated enough that the return from clear air above the cloud is too weak to be detected. In either case we refer to the "apparent cloud top". We have defined a transmittance index, $T_{\mathrm{i}}$, to help discriminate between true and apparent tops. $T_{\mathrm{i}}$ is equal to the fraction of the first 50 samples above the reported cloud top which are above background. If the signal has been completely attenuated, signal fluctuations are due only to noise in the background light level and $T_{\mathrm{i}}$ is near 0.5 . If $T_{\mathrm{i}}$ is near one, we can be confident a signal has been received from the clear air above the cloud.

The cloud-finding algorithm is applied to averaged lidar returns which are recorded every $15 \mathrm{~s}$. All cloud layers apparent in the return are identified, and base and top heights are tabulated with a resolution of 6,15 or $30 \mathrm{~m}$ (depending on sampling rate). Because of the short averaging time, many observations of a single cloud are recorded and the data may be used to investigate the structure of individual clouds. Each cloud detected in each 15-s average profile is considered a cloud observation.

Ground observers have been found to under-report the presence of altostratus and cirrus at night (Warren et al., 1985). However, a lidar system with marginal sensitivity will exhibit the opposite bias, being able to detect more high clouds at night than during the day, when noise due to skylight is higher. Fig. 3 shows scatter plots of the peak attenuated scattering ratio, $S R_{\mathrm{pk}}^{\prime}$, observed within cloud layers vs. the base height of the layer. Shown are data from ECLIPS Phase II for all layers above $8 \mathrm{~km}$ observed at night and observed within two hours of local noon, when sensitivity would be expected to be worst. Fig. 3a shows that at night cirrus layers with $S R_{\mathrm{pk}}^{\prime}$ as small as 3 are detected, while most cirrus layers have $S R_{\mathrm{pk}}^{\prime}$ between 10 and 100. Cirrus layers with $S R_{\mathrm{pk}}^{\prime}$ less than 10 to 15 have been found to be subvisible to a ground observer. In Fig. $3 \mathrm{~b}$ we find clouds with $S R_{\mathrm{pk}}^{\prime}$ as small as about 5 being detectable, allowing the great majority of cirrus layers to be detected. Differences shown in the two plots are due in part to diurnal variations in 

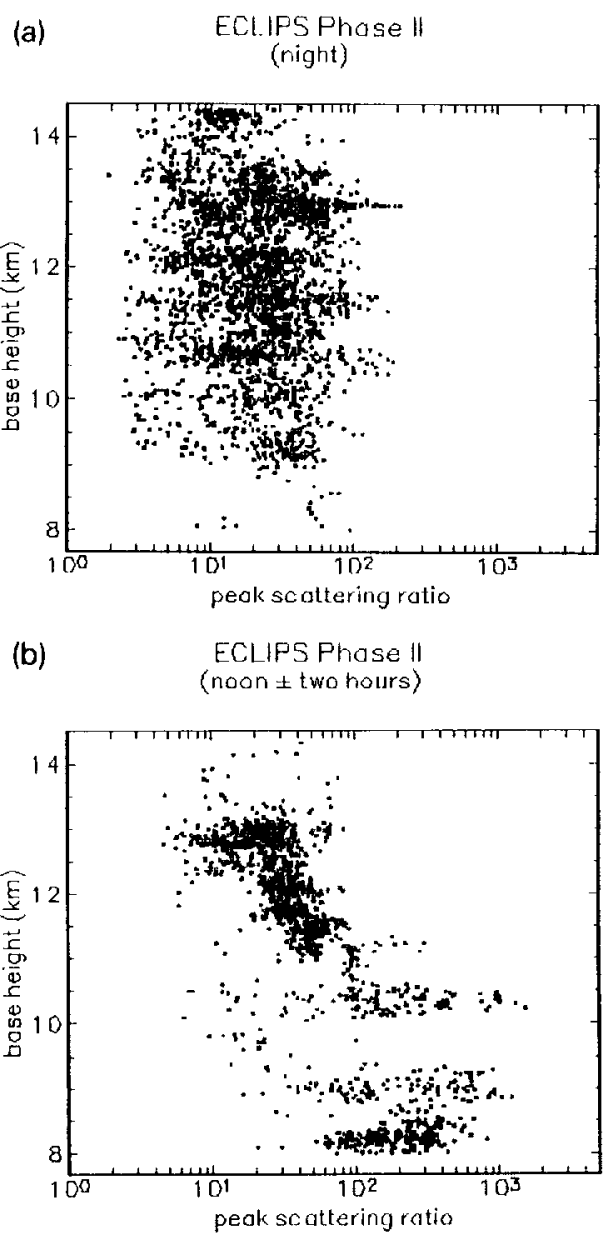

Fig. 3. Cloud observations from ECLIPS Phase II. ( (a) all nighttime observations above $8 \mathrm{~km}$; (b) all observations above $8 \mathrm{~km}$ and within 2 hours of solar noon.

cirrus occurrence, but Fig. 3b shows that the sensitivity limit of the system is nearly as good during the day as at night. This result is consistent with a sensitivity analysis which was performed, showing system sensitivity is limited more by digitizer quantization error than background noise.

\section{Cloud base height distributions}

Cloud base height is generally not observable from satellite instruments, therefore a primary objective of this work has been to observe base heights and derive statistics which might be used for parameterization of cloud base height both for retrievals and for climate modelling. As an example, Fig. 4 shows the frequency 

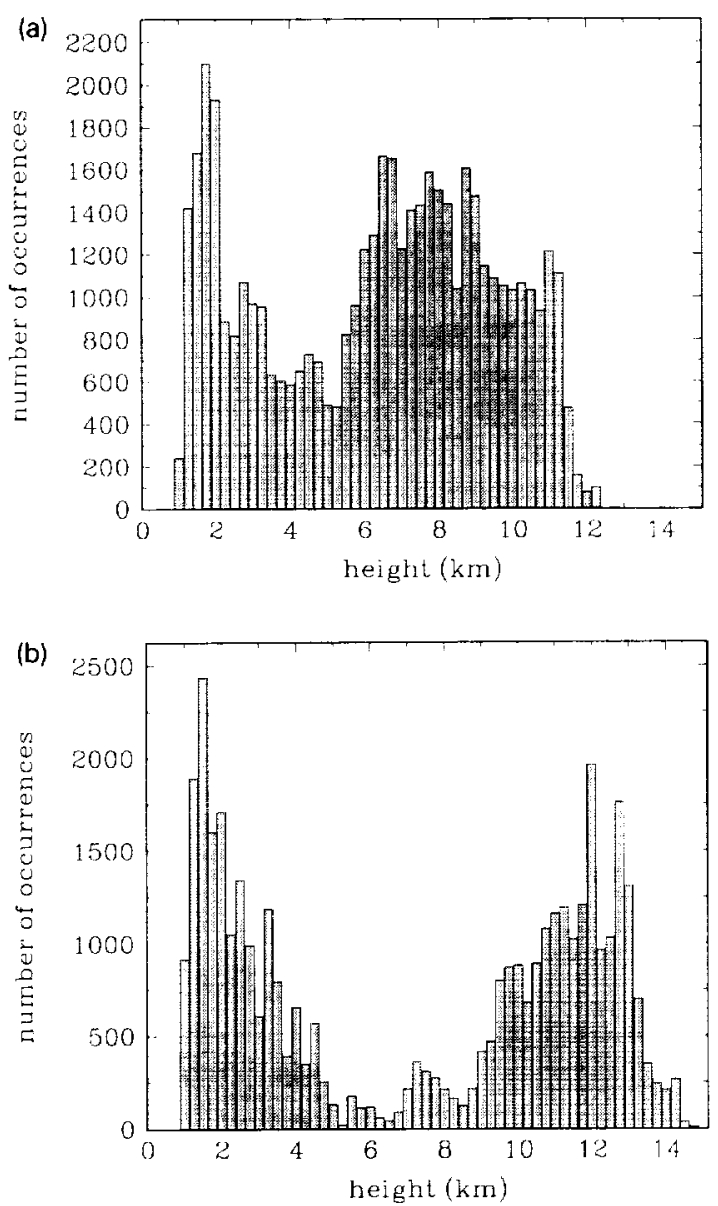

Fig. 4. Cloud base height distributions from ECLIPS. (a) Phase I, (b) Phase II.

of occurrence of clouds as a function of cloud base altitude for the two observation phases of ECLIPS. ECLIPS Phase I and Phase II can be used to represent cloud statistics for the fall and spring seasons, respectively. Fig. 5 shows statistics derived from FIRE ETO observations for the other two seasons. The ECLIPS histograms exhibit much less statistical fluctuation than the FIRE ETO histograms as they are based on about ten times as much observing time as the FIRE ETO histograms. The number of cloud observations going into each data set is given in Table 2. A total of about $1000 \mathrm{~h}$ of observations were conducted during the two phases of ECLIPS.

The lowest altitude at which the lidar system is able to detect clouds is about 1 $\mathrm{km}$. Lower base heights can be obtained from the ceilometer operated at the site but have not been included in this study. The average height for cirrus clouds follows the seasonal motion of the mean tropopause height. High cirrus are often 

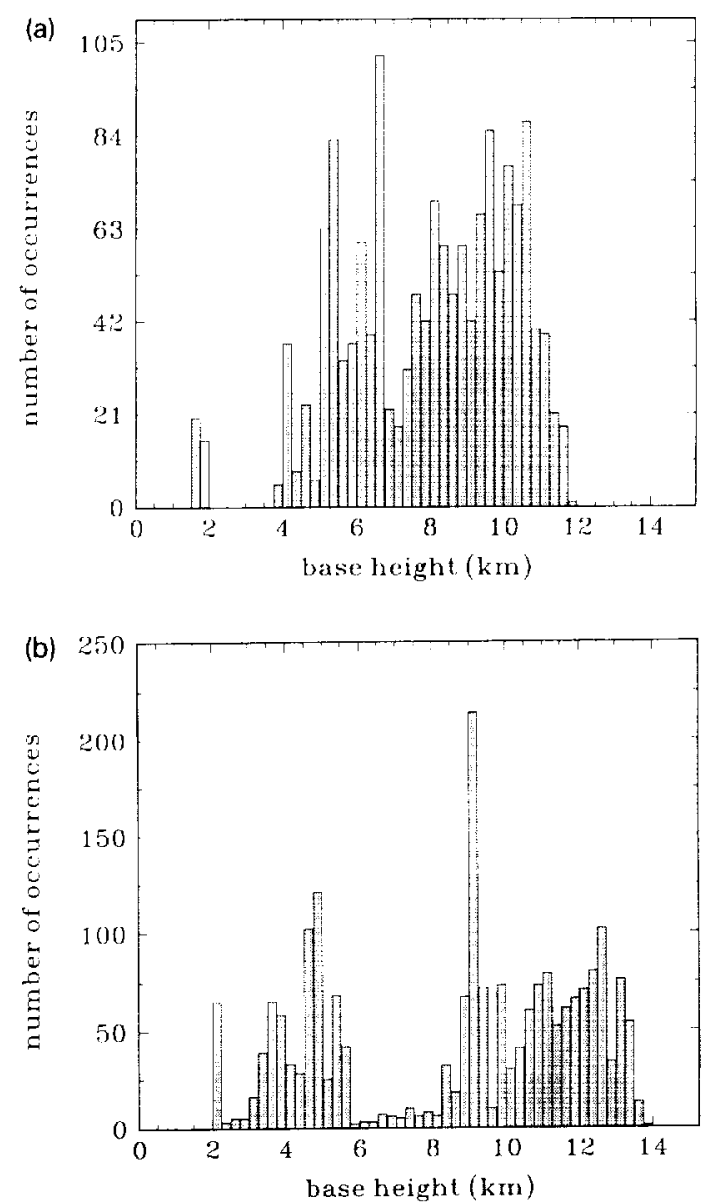

Fig. 5. Base height distributions from FIRE ETO. (a) Winter: January - March 1990, (b) Summer: July - September 1990.

Table 2

Number of cloud observations by season. Winter and summer 1990 are from the FIRE ETO program

Fall 1989 (ECLIPS I)

Winter 1990

Spring 1991 (ECLIPS II)

Summer 1990
47,740

1524

38,831

2110

very thin, so that cloud bases are observed up to the height of the tropopause. Fig. 6 shows mean temperature profiles for the two observation periods. The shift in tropopause height is consistent with the shift in maximum cloud base heights shown in Fig. 4.

The distribution of cloud base height appears to be bimodal, with a minimum 


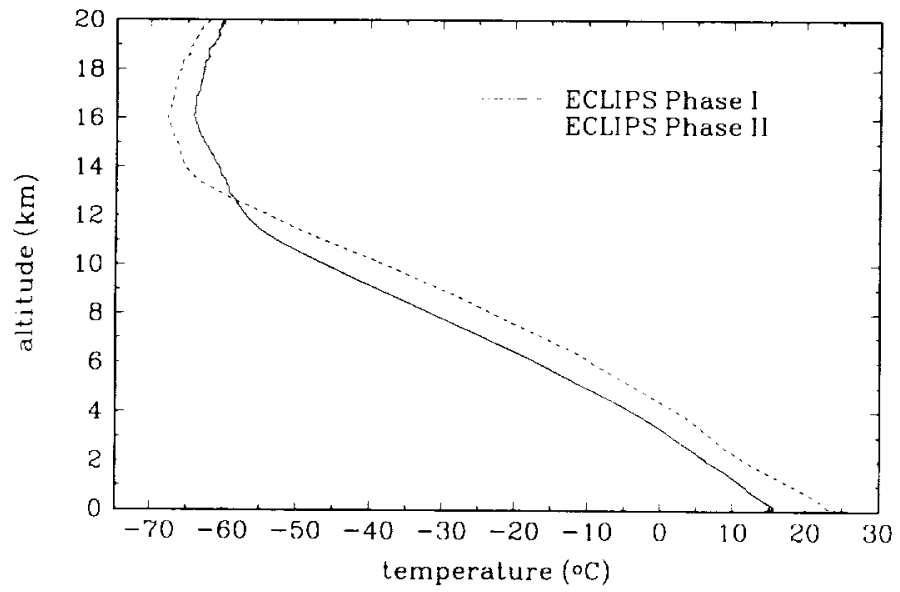

Fig. 6. Phase I and Phase II mean temperature profiles.

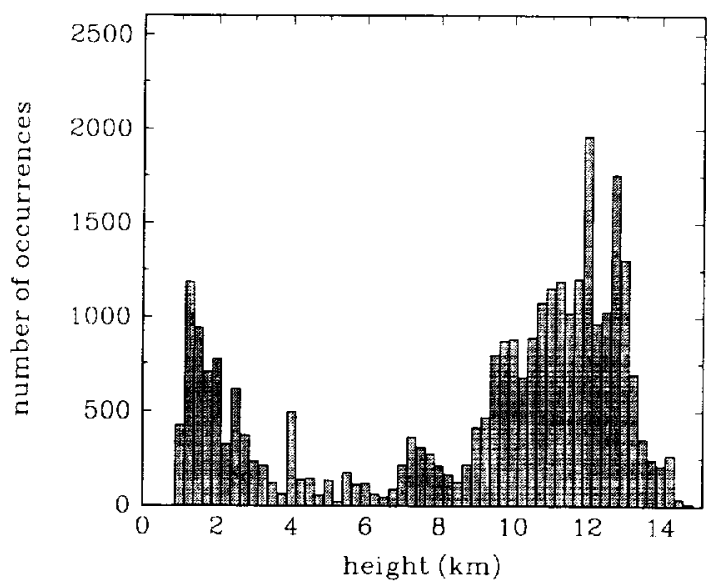

Fig. 7. Base height distributions during ECLIPS Phase II, low opaque clouds removed.

in the frequency of occurrence of cloud base in the 4 to $8 \mathrm{~km}$ region. The minimum occurs at a somewhat higher altitude in spring and summer than in winter. It is seen to be a persistent feature, though it is more evident in some of the seasonal datasets than in others. The possibility that this feature is an artifact, due to blockage of mid-level clouds by lower opaque clouds, was investigated. Fig. 7 was computed from the same set of profiles as Fig. 4a but with low opaque clouds removed. This dramatically reduces the occurrence of low clouds, but there are still more low transparent clouds than mid-level clouds of all types. The shape of the lower mode was shown in the data of deBary and Möller (1963), with cloud frequency decreasing above $4 \mathrm{~km}$. Their statistics were based on aircraft pilot reports, which extended only to an altitude of $5 \mathrm{~km}$. 
Profiles from rawindsondes flown during ECLIPS show the presence on many days of deep moist layers above the surface, capped by inversion layers. Moist layers as deep as $4 \mathrm{~km}$ are seen. The distribution of the depth of these moist layers qualitatively explains the decreasing occurrence of cloud base above $2 \mathrm{~km}$. The majority of clouds which are seen at mid-levels appear to be associated with frontal passages, and few clouds are observed if no frontal activity experienced on observation days. More frontal activity was experienced in Phase I than in Phase II, leading to a higher occurrence of cloud in this altitude region.

\section{Mid-cloud height and cloud thickness distributions}

We somewhat arbitrarily define low clouds as those which are mostly water, high clouds as those which are mostly ice, and mid- level clouds as those which
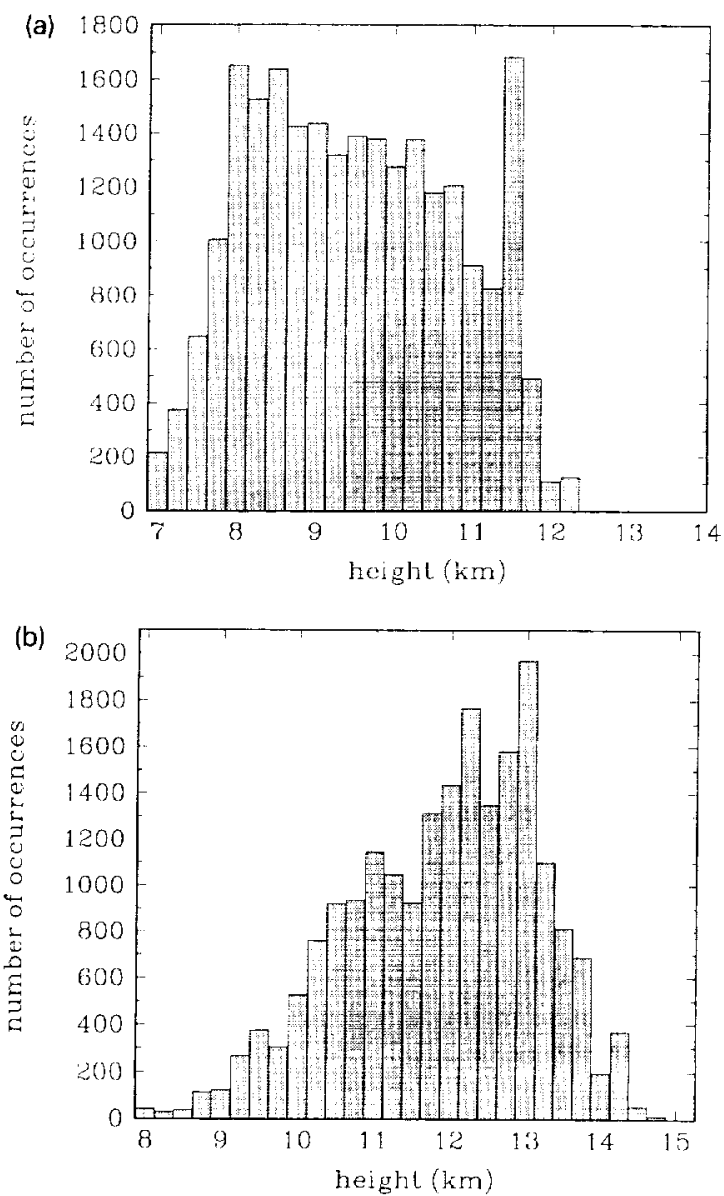

Fig. 8. Distribution of cirrus mid-cloud altitudes. (a) ECLIPS Phase I, (b) ECLIPS Phase II. 

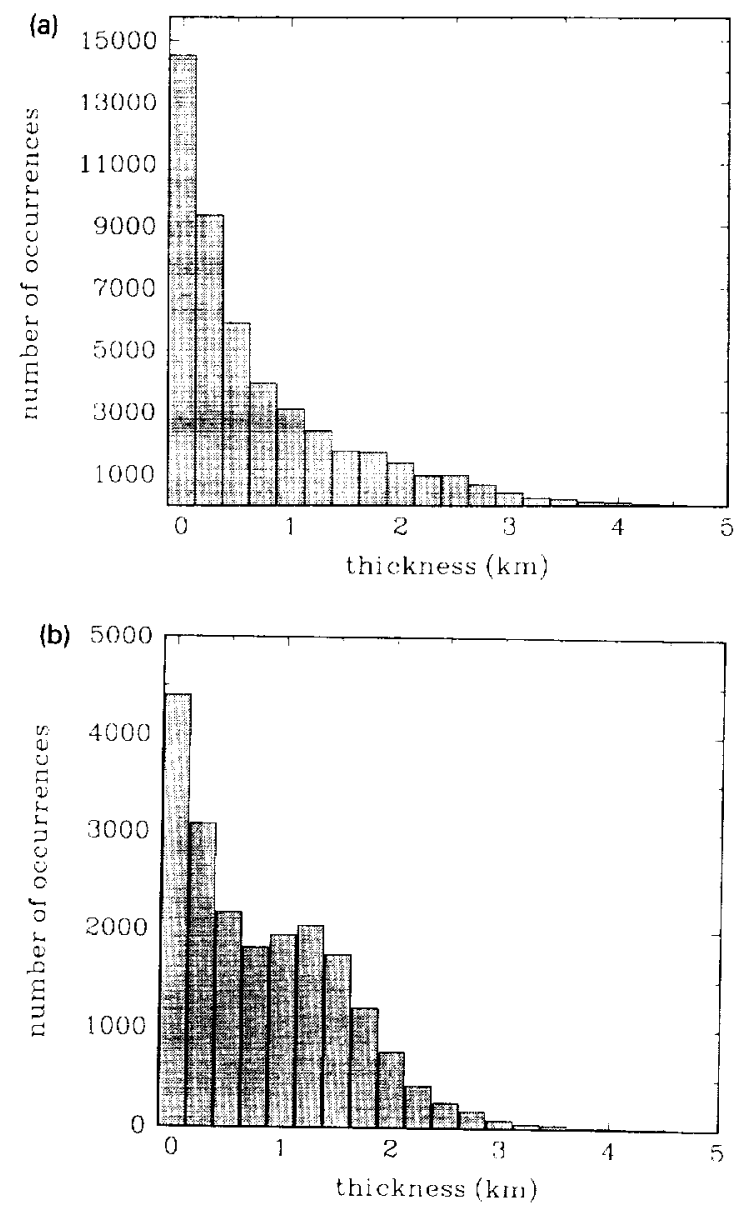

Fig. 9. Distribution of cirrus thickness. (a) ECLIPS Phase I, (b) ECLIPS Phase II.

are mixed-phase. At this time we have not based this classification on the lidar depolarization data. Rather, a crude classification has been performed based on the mean temperature profiles shown in Fig. 6, and fig. 2-1 of Prupacher and Klett (1980) showing the observed frequency of water and ice in clouds as a function of temperature. Using this approach, low clouds are defined as those with base heights less than 4 (5) $\mathrm{km}$ for ECLIPS Phase I (II). High clouds are defined as those with base heights greater than 7 (8) $\mathrm{km}$ for ECLIPS Phase I (II), and mid-level clouds are those in the intermediate ranges. The terms "high cloud" and "cirrus" are used interchangeably.

For cirrus clouds, attenuation is typically small and a lidar return from above the cloud is obtained in a majority of cases. Low clouds, on the other hand, are most often opaque, and the lidar pulse fails to penetrate the cloud. In this case we can report only an apparent top and can compute a penetration depth rather than 


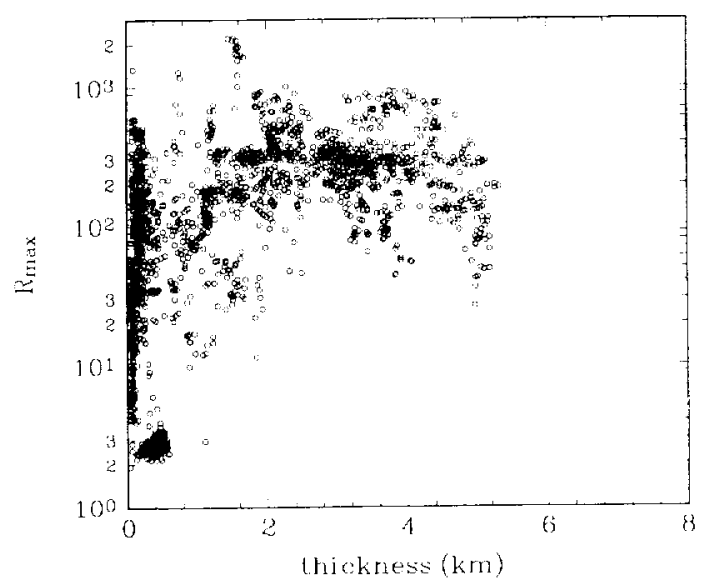

Fig. 10. Peak scattering ratio plotted as a function of cloud thickness for cloud bases between 4 and 7 $\mathrm{km}$.

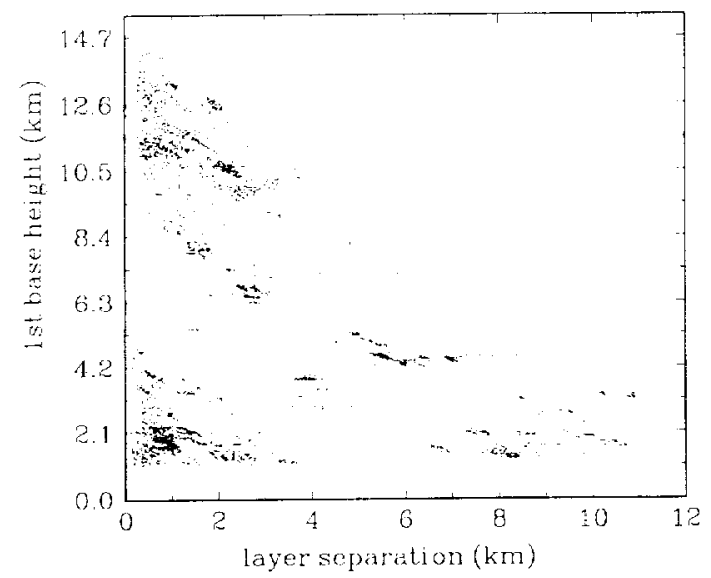

Fig. 11. Vertical separation of cloud layers vs. height of the lower cloud base.

cloud thickness. Because reported lidar cloud tops are not representative of true cloud top for low clouds, we do not discuss top height or thickness of low clouds.

Top height statistics for high clouds were computed using only clouds for which "true cloud top" heights were reported. A transmittance index criterion of $T_{\mathrm{i}}>0.8$ was used to discriminate between true and apparent tops. Top height statistics were computed again including all clouds, regardless of the value of $T_{i}$. The distributions in the two cases were nearly identical, indicating that in cases where an apparent top was reported, the lidar pulse was actually reaching the true cloud top. Once the validity of the reported cloud tops was established, mid-cloud heights were computed (defined as the average of cloud base height and top height). Fig. 
8 shows statistics of mid-cloud height corresponding to the cloud bases shown in Fig. 4. The shapes of the distributions are very similar to those for base height, but shifted upward by about $0.5 \mathrm{~km}$.

Fig. 9 shows the histogram of cirrus thickness derived from cloud base and top measurements for ECLIPS Phases I and II. Given the high spatial resolution of the lidar and sensitivity to tenuous cloud, a great many observations were made of very thin layers. The majority of cirrus were seen to be less than $1 \mathrm{~km}$ thick, though clouds more than $4 \mathrm{~km}$ deep were seen. It is not known if the difference in the shapes of the distributions is statistically significant.

Fig. 10 shows that two distinct types of mid-level cloud are seen. Water clouds are highly attenuating and therefore show a small apparent depth with a wide range of peak scattering ratios. Another class of cloud exhibits very high scattering and yet has measurable thicknesses of many kilometers. The exact morphology of these clouds is not known, but the depolarization signatures reveal that they are ice clouds. They seem to be associated with frontal systems, are initially generated at cirrus altitudes, and gradually decrease in altitude as the front moves through. The small cluster of points in the lower left corner arises not from a true cloud but a from a moist inversion layer where aerosols have begun to grow due to the high humidity.

The lidar often observes multiple layer clouds. About $13 \%$ of the lidar profiles include 2 or more layers and $1 \%$ include three or more layers. Fig. 11 shows the distribution of the spacing between multiple cloud layers observed in single lidar profiles. Most of the observations fall into three categories: data points in the lower left quadrant correspond to multilayer stratus, points in the upper left quadrant correspond to multilayer cirrus clouds, and points in the lower right quadrant correspond to the simultaneous observation of stratus and cirrus.

\section{Summary}

Table 3 summarizes the relative frequency of occurrence of low, mid-level and high clouds. Cirrus represented about half the cloud observations for both seasons, while large differences were seen in the occurrence of low and mid-level clouds. These differences are due to the greater synoptic-scale frontal storms experienced during Phase I, typical of that time of year. The number of hours of cirrus observed as a percentage of the total number of hours of observations per-

Table 3

Frequency of occurrence of clouds as a fraction of the total number of cloud observations

\begin{tabular}{lcr}
\hline & Phase I & Phase II \\
\hline$f($ high $)$ & $48 \%$ & $52 \%$ \\
$f($ mid $)$ & $23 \%$ & $5 \%$ \\
$f($ low $)$ & $28 \%$ & $43 \%$ \\
\hline
\end{tabular}


Table 4

Statistics of observed cirrus mid-cloud altitude and thickness

\begin{tabular}{|c|c|c|}
\hline & Phase I & Phase II \\
\hline \multicolumn{3}{|c|}{ Mid-cloud altitude: } \\
\hline range & $7-12.9 \mathrm{~km}$ & $8-14.9 \mathrm{~km}$ \\
\hline mean & $9.6 \mathrm{~km}$ & $12.0 \mathrm{~km}$ \\
\hline st. dev. & $1.3 \mathrm{~km}$ & $\begin{array}{r}1.25 \mathrm{~km} \\
\text { thickness: }\end{array}$ \\
\hline range & $0.03-4.3 \mathrm{~km}$ & $0.03-4.5 \mathrm{~km}$ \\
\hline mean & $0.86 \mathrm{~km}$ & $0.94 \mathrm{~km}$ \\
\hline st. dev. & $0.76 \mathrm{~km}$ & $0.74 \mathrm{~km}$ \\
\hline
\end{tabular}

formed was $18 \%$ for Phase I and $19 \%$ for Phase II. We have not tried to correct the percentages of low cloud occurrence for stratus which occurred below $1 \mathrm{~km}$ and was not detected by the lidar system. Neither have we tried to correct the percentages of mid-level and cirrus cloud occurrence for periods of stratus overcast when the lidar was unable to penetrate the low cloud layer. While some research indicates the probability of mid-and high-cloud occurrence to be independent of the presence of stratus (Hahn et al., 1982), this has not been well established. This issue will be a subject of future investigations.

Table 4 summarizes statistics of the mid-cloud height and thickness for high clouds. The mean mid-cloud altitude is seen to be about $80 \%$ of the mean tropopause height. The minimum altitudes listed result from the definition adopted for high clouds: clouds with significant ice content were seen at much lower altitudes. Clear distinctions between cirrus and alto-level clouds based solely on lidar signatures were generally not possible. The minimum thickness of $30 \mathrm{~m}$ represents the resolution limit of the lidar. The mean thickness observed is significantly smaller than the $1.5 \mathrm{~km}$ given by Dowling and Radke (1990). This may be because the studies summarized by Dowling and Radke were more in the nature of case studies than continuous monitoring programs and may have concentrated on thicker, more obvious cloud systems.

It is hoped this study will contribute to the development of climatologies of cloud characteristics. The data presented here is part of a database which has been compiled at NASA Langley Research Center and which is being augmented by new cloud observations as the data is analyzed and becomes available. The data will eventually be submitted to the FIRE and ECLIPS data archives and become available to the community at large. In the meantime, the data can be made available on a collaborative basis by contacting the authors.

\section{Acknowledgement}

We would like to recognize the efforts of Joe Alvarez, who developed the lidar system used in making these observations and took much of the ECLIPS data analyzed here. 


\section{Appendix - cloud base and top algorithm}

The cloud-finding algorithm operates on attenuated scattering ratios. The raw signal profiles, $P(z)$, are first normalized using an experimentally determined estimate of the lidar system calibration constant, $C$, to produce a profile of attenuated backscatter:

$$
\beta^{\prime}(z)=\frac{z^{2} P(z)}{C}
$$

The profile of attenuated backscatter corresponding to a molecular atmosphere, $\beta_{\mathrm{m}}^{\prime}(z)$, is then estimated using an atmospheric model and is used to compute a profile of attenuated scattering ratio:

$$
S R^{\prime}(z)=\beta^{\prime}(z) / C \beta_{\mathrm{m}}^{\prime}(z),
$$

In the absence of noise, clouds could be identified as those regions of the return profile where the scattering ratios consistently exceed a threshold level of 1 . In practice, noise in the data is always significant at high altitudes, where cirrus are found, and often at lower altitudes also. To avoid noise spikes being identified as clouds, the minimum scattering ratio threshold for cloud determination is generally set to some number greater than unity.

In calculating scattering ratios, the raw signal profiles are divided by $\beta_{\mathrm{m}}^{\prime}(z)$ which is a smooth, decreasing function of altitude. As a consequence, noise in the data is amplified increasingly with altitude. Therefore instead of a constant threshold we use an altitude-dependent threshold of the form:

$$
\operatorname{SRT}(z)=1+\operatorname{SRT}\left(z_{\mathrm{o}}\right) / \beta_{\mathrm{m}}^{\prime}(z)
$$

which increases monotonically with altitude. The constant $\operatorname{SRT}\left(z_{0}\right)$ depends on the background noise level in the lidar signal. It is chosen at an altitude near the top of the profile and is set, somewhat arbitrarily, so as to provide discrimination between clouds and noise in the profile. This algorithm has been found to be a highly effective filter of noise at high altitudes. At low altitudes, however, it often becomes too small. In these regions a constant threshold value is used. The resulting threshold value for a given data point at height $z$ is the larger of $\operatorname{SRT}(z)$ and the minimum scattering ratio threshold.

To be recognized as a cloud base, a candidate feature must then exceed the threshold value for a certain number of consecutive points. A lower limit of seven consecutive points has been found to work well in the vast majority of cases. This algorithm fails to detect extremely thin clouds, however. Certain altostratus and altocumulus clouds have been observed which are very thin but exhibit high scattering. Therefore each profile failing the first test is tested according to a second criterion: a cloud is identified if for any three consecutive points exceeding their threshold levels, at least one is greater than some "high intensity" threshold.

Cloud top height is determined iteratively. An initial estimate is made at the first point above cloud base for which 4 or more of the next 7 points are at or below the threshold level. We then use our cloud base algorithm to scan a prede- 
fined altitude range above the initial estimate. If no secondary cloud base appears in this region, the initial cloud top altitude is confirmed. If, on the other hand, a new feature is found, the cloud top estimate is revised upward to the new cloud base, and the process is repeated. The algorithm continues in this fashion until eventually reaching a sufficiently clear region above the final cloud top estimate.

\section{References}

Cox, S.K., McDougal, D.S., Randall, D.A. and Schiffer, R.A., 1987. FIRE - The first ISCCP regional experiment. Bull. Am. Meteorol. Soc., 68: 114-118.

deBary, E. and Möller, F., 1963. The vertical distribution of clouds. J. Appl. Meteorol., 2: 806-808.

Dowling, D.R. and Radke, L.F., 1990. A summary of the physical properties of cirrus clouds. J. Appl. Meteorol., 29: 970-978.

Hahn, C.J., Warren, S.G., London, J., Chervin, R.M. and Jenne, R., 1982. Atlas of Simultaneous Occurrence of Different Cloud Types over the Ocean. NCAR TN-201+ STR.

Hahn, C.J., Warren, S.G., London, J., Chervin, R.M. and Jenne, R., 1984. Atlas of Simultaneous Occurrence of Different Cloud Types over Land. NCAR TN-242 + STR.

Minnis, P., Heck, P.W. and Harrison, E.F., 1990. The 27-28 October 1986 FIRE IFO cirrus case study: Cloud parameter fields derived from satellite data. Mon. Weather Rev., 11 8: 2426-2446.

Platt. C.M.R.. 1979. Remote sounding of high clouds: I. Calculation of visible and infrared optical properties from lidar and radiometer measurements. J. Appl. Meteorol., 18: 1130-1143.

Prupacher, H.R, and Klett, J.D., 1980. Microphysics of Clouds and Precipitation. Reidel, Boston, $714 \mathrm{pp}$.

Ramanathan, V., Cess, R.D., Harrison, E.F., Minnis, P., Barkstrom, B.R., Ahmad, E. and Hartmann, D., 1989. Cloud-radiative forcing and climate: Results from the Earth Radiation Budget Experiment. Science, 243: 57-63.

Rossow, W.B. and Schiffer, R.A., 1991. ISCCP cloud data products. Bull. Am. Meteorol. Soc., 72: 220.

Russell, P.B., Swissler, T.J. and McCormick, M.P., 1979. Methodology for error analysis and simulation of lidar aerosol measurements. Appl. Opt., 18: 3783-3797.

Warren, S.G., Hahn, C.J. and London, J., 1985. Simultancous occurrence of different cloud types. J. Clim. Appl. Meteorol., 24: 658-667.

WMO, 1988. An Experimental Cloud Lidar Pilot Study (ECLIPS): Report of the WCRP/CSIRO workshop on cloud base measurements. (CSIRO, Mordialloc, Victoria, Australia, February 29 March 3, 1988), WCRP-14, WMO/TD-No. 251, WMO, Geneva, Sept.

Wylic, D.P. and Menzel, W.P., 1989. Two years of cloud cover statistics using VAS. J. Climate, 2: 380-392. 
